\title{
Entrecomp: marco competencial para el emprendimiento. Una revisión sistemática de la literatura sobre su uso y aplicación
}

\author{
Pedro Baena-Luna(1), Esther García-Río(1) y Mauricio Monge-Agüero(2) \\ (1) Departamento de Administración de Empresas y Marketing, Universidad de Sevilla, Sevilla-España. \\ (correo-e: pbaenaluna@us.es) \\ (2) Instituto Tecnológico de Costa Rica. Cartago-Costa Rica.
}

Recibido Ago. 14, 2019; Aceptado Oct. 14, 2019; Versión final Dic. 9, 2019, Publicado Abr. 2020

\section{Resumen}

A partir de la revisión de la literatura de carácter científico relacionada con el marco competencial para el emprendimiento Entrecomp, se analiza y discute sobre el uso y el nivel de aplicación que ha tenido, desde su publicación en 2016, en la producción científica relacionada con las competencias emprendedoras y su entrenamiento. Entrecomp, surge a instancias de la Comisión Europea, con el objetivo de establecer una herramienta que pudiera ser utilizada a la hora de analizar la orientación emprendedora de los planes de formación y estudios de los países que conforman la Unión Europea. La metodología empleada ha sido la de la revisión sistemática de los trabajos relacionados con las competencias emprendedoras y de manera más específica con el marco competencia Entrecomp. Los resultados obtenidos permiten determinar el escaso impacto y limitado uso que ha tenido hasta ahora este marco competencial. Esto se observa tanto en trabajos de carácter empírico como de carácter teórico.

Palabras clave: Entrecomp; emprendimiento; competencias emprendedoras; educación emprendedora

\section{Entrecomp: competent framework for entrepreneurship. A systematic review of the literature on its use and application}

\begin{abstract}
From a review of the scientific literature related to the competence framework for Entrecomp entrepreneurship, aspects on the use and level of application that Entrecomp has had since its publication in 2016, in scientific production related to entrepreneurial skills and their training have been analyzed and discussed. Entrecomp, arises at the request of the European Commission, with the aim of establishing a tool that could be used when analyzing the entrepreneurial orientation of the training and study plans of the countries that form the European Union. The methodology used has been the systematic review of the work related to entrepreneurial competencies and more specifically with the Entrecomp competence framework. The results obtained allow determining the low impact and limited use that this competency framework has had until now. This has been observed for both empirical and theoretical works.
\end{abstract}

Keywords: Entrecomp; entrepreneurship; entrepreneurship competencies; entrepreneurial education 


\section{INTRODUCCIÓN}

El fenómeno del emprendimiento es una realidad en auge (Soria-Barreto et al., 2016). Se ha conformado como una piedra angular del crecimiento económico de los territorios (Rengamani y Shameem, 2018). Una economía en constante evolución como la actual (Sanchis et al., 2009), favorece la aparición de nuevas actividades económicas y aumenta el papel del emprendimiento como elemento clave para la generación de empleo y el crecimiento económico (Davidsson, 2015). Es un concepto complejo que abarca desde la generación de ideas, hasta el proceso de puesta en marcha de éstas. Esto da lugar a conexiones con otros ámbitos de la sociedad (Jiménez et al., 2015). El emprendimiento influye no solo en la actividad económica. Está presente en otros ámbitos como el social, el personal y el educativo (Paños-Castro, 2017).

De acuerdo con Rengamani y Shameem (2018), a través de una iniciativa emprendedora, la persona lleva a cabo la aplicación una serie de conocimientos, habilidades y competencias de manera que le lleven a la consecución del objetivo perseguido. Fagerholm et al. (2018) señala como, a través de trabajos previos, se ha podido constatar cómo los programas educativos y capacitadores, influyen de manera positiva en el favorecimiento del espíritu emprendedor. En el caso de las competencias relacionadas con la actividad emprendedora, existe un gran espectro de rasgos y características (Aurik y Astri, 2018). Uno de los principales obstáculos para el desarrollo de una mentalidad emprendedora y sus competencias es que, de manera similar al espíritu emprendedor, la innovación y la creatividad se perciben (y son considerados) con frecuencia, como rasgos y no como algo que puede ser desarrollados (Edwards-Schachter et al., 2015). A pesar de ser vitales para el rendimiento empresarial, los estudios de competencias para el emprendimiento están todavía en una etapa preliminar (Aurik y Astri, 2018).

El estudio y desarrollo de las competencias emprendedoras se encuentra ante distintos retos por superar. Existe una gran diversidad de opiniones confusas, conceptualizaciones e interpretaciones (EdwardsSchachter et al., 2015). "El emprendimiento ha llegado a ser una etiqueta en la que se han incluido una gran cantidad de investigación multidimensional" (Palos-Sánchez et al. 2019). El espíritu emprendedor puede enseñarse y las metodologías aplicadas pueden mejorarse mediante la participación activa (Fagerholm et al., 2018). Las personas emprendedoras descubren las oportunidades donde otros no las ven (Suddaby et al., 2015). Ante esto, la capacitación, les proporciona habilidades cognitivas para evaluar y explotar mejor las oportunidades empresariales, aumentar el nivel de confianza en sí mismos y reducir el riesgo percibido (Jiménez et al., 2015).

Es habitual en la actualidad, que los actores políticos consideren que la educación para el emprendimiento tiene un gran potencial para que los jóvenes adquieran habilidades y generen sus propios trabajos cualificados (Premand et al., 2016). En el caso de la UE, desde el año 2014, la educación emprendedora ocupa un lugar relevante dentro de sus acciones. Es una dirección estratégica dentro de sus políticas educativas. Ya en el año 2000, el Consejo Europeo de Lisboa había establecido el objetivo de transformar la productividad de los territorios, a través de la creación de una cultura emprendedora y de innovación. Más adelante, en 2006, el Parlamento Europeo especificó que las habilidades emprendedoras eran competencias clave para el aprendizaje permanente. Finalmente, en 2016, la Comisión Europea estableció Entrecomp en el trabajo de Bacigalupo et al. (2016). Este marco establece una herramienta que posibilita analizar la orientación emprendedora de los planes de formación y estudios en la UE (Strauti et al., 2018).

En este trabajo, se lleva a cabo una revisión del impacto en la literatura científica del uso y aplicación del marco competencial emprendedor Entrecomp en la producción científica relacionada con las competencias para el emprendimiento y su entrenamiento. Para ello se han analizado las principales bases de datos de carácter científico. Fruto de esta revisión ha sido la constatación del limitado impacto y la escasa aplicación que ha tenido Entrecomp, dentro de la literatura relacionada, desde su publicación.

\section{OTROS ANTECEDENTES}

Entrecomp define al emprendimiento como una competencia universal aplicable a distintos ámbitos (Aurik y Astri, 2018) y esencial en un mercado de trabajo como el actual (Lynch et al., 2019). La UE pretende crear un puente entre la educación y las acciones que tengan por objeto favorecer al fomento del espíritu emprendedor (Bacigalupo et al., 2016). Entrecomp parte de una de una definición de espíritu emprendedor caracterizada como competencia. Éste es definido como "la capacidad de actuar sobre las oportunidades e ideas y así crear valor para los demás. El valor obtenido puede ser social, cultural o financiero" (McCallum et al. 2018). En el proceso de conformación de este marco competencial, se integraron no solo la visión academicista del fenómeno del emprendimiento. Como puede observarse en la figura 1 a lo largo de éste, fueron varios los agentes implicados y tomados en cuenta antes de establecer el marco competencial para el emprendimiento Entrecomp. 


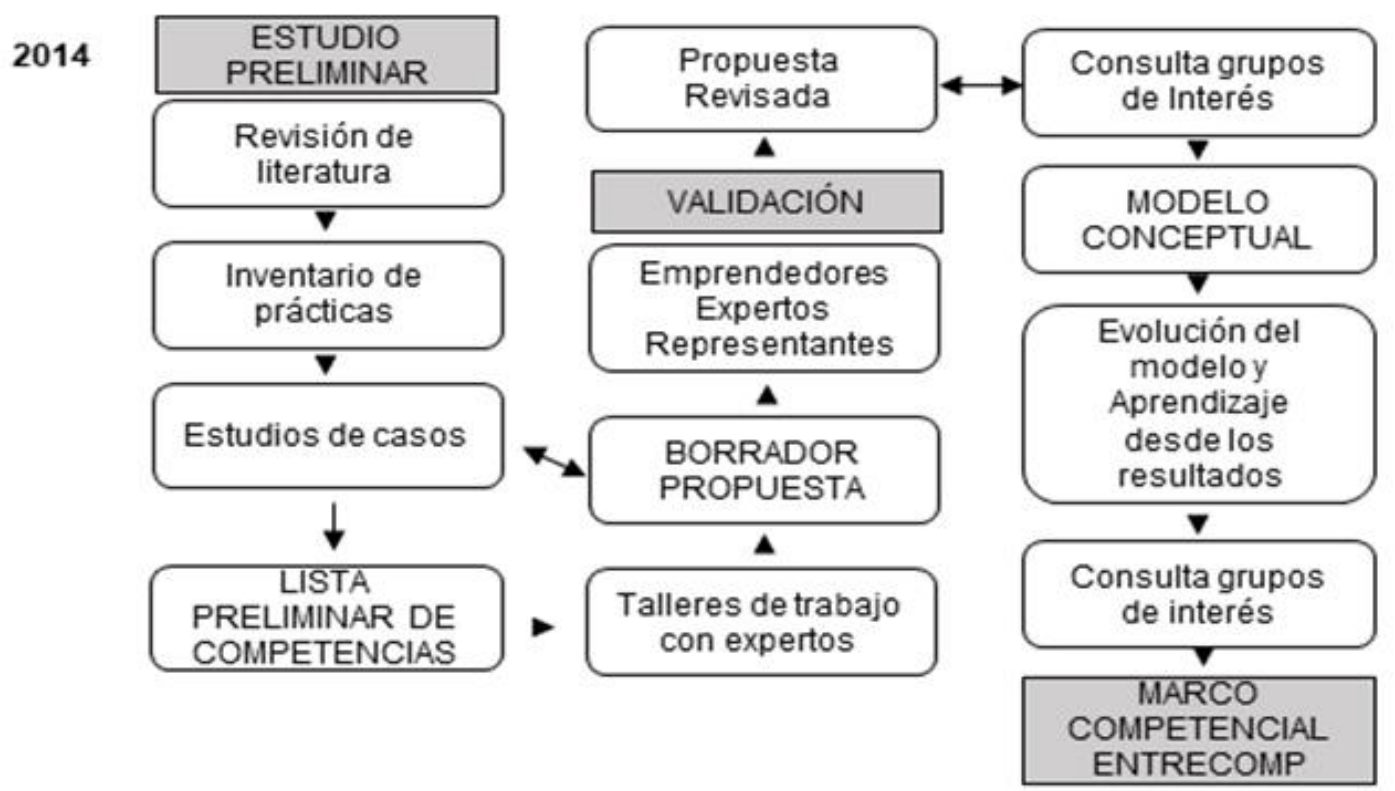

Fig. 1. Diagrama de flujo sobre las etapas del marco competencial Entrecomp (adaptada de Bacigalupo et al. 2016)

El modelo propuesto en Entrecomp, como se observa en la tabla 1, tiene 3 áreas competenciales. Éstas a su vez se encuentran conformadas por 15 competencias específicas. Todas ellas incluyen una serie de consejos y recomendaciones junto a unos descriptores. También contiene un modelo de progresión dividido en 8 niveles dando lugar a cuatrocientos 42 resultados esperados de aprendizaje. Según sus autores, Entrecomp, "refleja la complejidad del dominio de competencia emprendedora, que aborda varios aspectos de nuestra vida cotidiana y se puede utilizar como una guía de referencia de múltiples propósitos."(Bacigalupo et al., 2016; p.14).

Tabla 1. Áreas competenciales y competencias específicas Entrecomp. (Bacigalupo et al., 2016)

\begin{tabular}{|c|c|c|c|}
\hline Áreas & Competencias & Recomendaciones & Descriptores \\
\hline \multirow{5}{*}{ 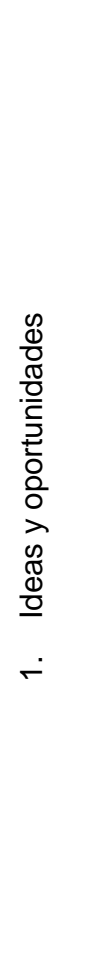 } & $\begin{array}{l}\text { Identificar } \\
\text { oportunidades }\end{array}$ & $\begin{array}{l}\text { Usa tu imaginación y } \\
\text { habilidades para } \\
\text { identificar oportunidades y } \\
\text { crear valor. }\end{array}$ & $\begin{array}{l}\text { Identifique y aproveche las oportunidades para crear valor } \\
\text { explorando el panorama social, cultural y económico / } \\
\text { Identifique las necesidades y los desafíos que deben } \\
\text { cumplirse / Establezca nuevas conexiones y reúna a los } \\
\text { elementos del paisaje para crear oportunidades para crear } \\
\text { valor. }\end{array}$ \\
\hline & Creatividad & $\begin{array}{l}\text { Desarrollar ideas } \\
\text { creativas y útiles. }\end{array}$ & $\begin{array}{l}\text { Desarrolle varias ideas y oportunidades para crear valor, } \\
\text { incluidas mejores las soluciones a los desafíos nuevos y } \\
\text { existentes / Explore y experimente con enfoques } \\
\text { innovadores / Combine conocimientos y recursos para } \\
\text { lograr efectos valiosos. }\end{array}$ \\
\hline & Visión & $\begin{array}{l}\text { Trabajar con una visión } \\
\text { de futuro. }\end{array}$ & $\begin{array}{l}\text { Imagine el futuro / Desarrolle una visión para convertir } \\
\text { ideas en acción / Visualice escenarios futuros para ayudar } \\
\text { a guiar el esfuerzo y la acción. }\end{array}$ \\
\hline & Evaluar ideas & $\begin{array}{l}\text { Aprovecha al máximo las } \\
\text { ideas y oportunidades. }\end{array}$ & $\begin{array}{l}\text { Juzgue qué valor tiene en términos sociales, culturales y } \\
\text { económicos / Reconozca el potencial que tiene una idea } \\
\text { para crear valor e identifique formas adecuadas de } \\
\text { aprovecharla al máximo. }\end{array}$ \\
\hline & $\begin{array}{l}\text { Pensamiento } \\
\text { ético y } \\
\text { sostenible }\end{array}$ & $\begin{array}{l}\text { Evaluar las } \\
\text { consecuencias y el } \\
\text { impacto de ideas, } \\
\text { oportunidades y acciones. }\end{array}$ & $\begin{array}{l}\text { Evalúe las consecuencias de las ideas que aportan valor y } \\
\text { el efecto de la acción empresarial en la comunidad } \\
\text { objetivo, el mercado, la sociedad y el medio ambiente. / } \\
\text { Reflexionar sobre cómo son los objetivos sociales, } \\
\text { culturales y económicos sostenibles a largo plazo, y el } \\
\text { curso de acción elegido / Actuar responsablemente. }\end{array}$ \\
\hline
\end{tabular}


Tabla 1: continuación

\begin{tabular}{|c|c|c|c|}
\hline Áreas & Competencias & Recomendaciones & Descriptores \\
\hline \multirow{5}{*}{ 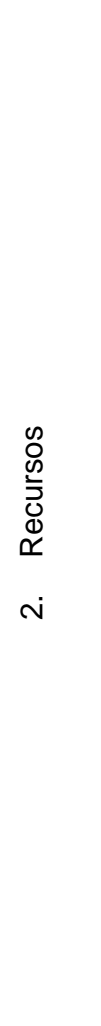 } & $\begin{array}{l}\text { Autoconocimiento y } \\
\text { confianza } \\
\text { en si mismo }\end{array}$ & $\begin{array}{l}\text { Creer en ti y seguir } \\
\text { desarrollándose. }\end{array}$ & $\begin{array}{l}\text { Reflexione sobre sus necesidades, aspiraciones y } \\
\text { deseos a corto, mediano y largo plazo / Identifique y } \\
\text { evalúe las fortalezas y debilidades individuales y } \\
\text { grupales / Cree en su capacidad para influir en el curso } \\
\text { de los eventos, a pesar de la incertidumbre, los } \\
\text { contratiempos y las fallas temporales. }\end{array}$ \\
\hline & $\begin{array}{l}\text { Motivación y } \\
\text { perseverancia }\end{array}$ & $\begin{array}{l}\text { Mantener la } \\
\text { concentración y no } \\
\text { darse por vencido. }\end{array}$ & $\begin{array}{l}\text { Esté determinado a convertir ideas en acción y } \\
\text { satisfacer su necesidad de lograr. / Esté preparado para } \\
\text { ser paciente y seguir intentando alcanzar sus objetivos } \\
\text { individuales o grupales a largo plazo. / Ser resistente } \\
\text { bajo presión, adversidad y falla temporal. }\end{array}$ \\
\hline & Movilizar recursos & $\begin{array}{l}\text { Recopilar y gestionar } \\
\text { los Recursos que se } \\
\text { necesitan. }\end{array}$ & $\begin{array}{l}\text { Obtenga y administre los recursos materiales, no } \\
\text { materiales y digitales necesarios para convertir ideas en } \\
\text { acción / Aproveche al máximo los recursos limitados / } \\
\text { Obtenga y administre las competencias necesarias en } \\
\text { cualquier etapa, incluidas las competencias técnicas, } \\
\text { legales, fiscales y digitales }\end{array}$ \\
\hline & $\begin{array}{l}\text { Educación financiera } \\
\text { y económica }\end{array}$ & $\begin{array}{l}\text { Desarrollar } \\
\text { conocimientos } \\
\text { financieros y } \\
\text { económicos. }\end{array}$ & $\begin{array}{l}\text { Estime el costo de convertir una idea en una actividad } \\
\text { de creación de valor / Planifique, aplique y evalúe las } \\
\text { decisiones financieras a lo largo del tiempo / Administre } \\
\text { los recursos financieros para asegurarse de que mi } \\
\text { actividad de creación de valor pueda durar a largo plazo }\end{array}$ \\
\hline & $\begin{array}{l}\text { Movilizar a otras } \\
\text { personas }\end{array}$ & $\begin{array}{l}\text { Inspirar, entusiasmar y } \\
\text { atraer a otras personas } \\
\text { a bordo. }\end{array}$ & $\begin{array}{l}\text { Inspire y entusiasme a las partes interesadas relevantes } \\
\text { / Obtenga el apoyo necesario para lograr resultados } \\
\text { valiosos / Demuestre comunicación, persuasión, } \\
\text { negociación y liderazgo efectivos }\end{array}$ \\
\hline \multirow{5}{*}{ 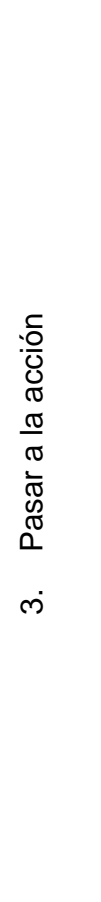 } & Tomar la iniciativa & Ir a por ello. & $\begin{array}{l}\text { Iniciar procesos que creen valor / asumir retos. / Actuar } \\
\text { y trabajar de forma independiente para lograr los } \\
\text { objetivos, atenerse a las intenciones y llevar a cabo las } \\
\text { tareas planificadas. }\end{array}$ \\
\hline & $\begin{array}{l}\text { Planificación y } \\
\text { gestión }\end{array}$ & $\begin{array}{l}\text { Priorizar, organizar y } \\
\text { hacer seguimiento. }\end{array}$ & $\begin{array}{l}\text { Establezca metas a largo, mediano y corto plazo / } \\
\text { Defina prioridades y planes de acción / Adáptese a } \\
\text { cambios imprevistos }\end{array}$ \\
\hline & $\begin{array}{l}\text { Manejar la } \\
\text { incertidumbre, la } \\
\text { ambigüedad y el } \\
\text { riesgo }\end{array}$ & $\begin{array}{l}\text { Tomar decisiones frente } \\
\text { a la incertidumbre, la } \\
\text { ambigüedad y el riesgo }\end{array}$ & $\begin{array}{l}\text { Tome decisiones cuando el resultado de esa decisión } \\
\text { sea incierto, cuando la información disponible sea } \\
\text { parcial o ambigua, o cuando exista un riesgo de } \\
\text { resultados no deseados / Dentro del proceso de } \\
\text { creación de valor, incluya formas estructuradas de } \\
\text { probar ideas y prototipos desde las etapas iniciales. } \\
\text { para reducir los riesgos de fallar / Manejar situaciones } \\
\text { de rápido movimiento de manera rápida y flexible }\end{array}$ \\
\hline & $\begin{array}{l}\text { Trabajar con otras } \\
\text { personas }\end{array}$ & $\begin{array}{l}\text { Trabajar y colaborar en } \\
\text { red. }\end{array}$ & $\begin{array}{l}\text { Trabaje junto con otros y coopere para desarrollar ideas } \\
\text { y convertirlas en acción / Red / Resolver conflictos y } \\
\text { enfrentar la competencia de manera positiva cuando } \\
\text { sea necesario }\end{array}$ \\
\hline & $\begin{array}{l}\text { Aprender de la } \\
\text { experiencia }\end{array}$ & Aprender haciendo & $\begin{array}{l}\text { Use cualquier iniciativa para la creación de valor como } \\
\text { una oportunidad de aprendizaje / Aprenda con otros, } \\
\text { incluyendo compañeros y mentores / Reflexione y } \\
\text { aprenda del éxito y el fracaso }\end{array}$ \\
\hline
\end{tabular}

Las 3 áreas competenciales: ideas y oportunidades, recursos y pasar a la acción reflejan la posibilidad de convertir ideas en acciones que generen valor no necesariamente en la propia persona. Las competencias específicas, un total de 15, muestran cuales son las que hacen emprendedora a una persona y que pueden ser utilizadas en distintos ámbitos (McCallum et al., 2018). De cada competencia específica, surgen una serie de hilos que vienen a explicar que supone a efectos prácticos cada una de ellas. Estos hilos se asocian a su vez a 8 niveles de progresión en los que puede estar la persona. Ésta puede obtener distintos resultados de aprendizaje fundamentados en 4 niveles: iniciales, intermedios, avanzados y expertos (Bacigalupo et al., 2016; McCallum et al., 2018). Este marco competencial favorece a la consideración del emprendimiento como una realidad que se puede aprender y enseñar y por tanto no limitado éste a la creencia de que a ser emprendedor no puede aprenderse ya que esta es una competencia que se posee o no. 


\section{METODOLOGÍA}

La técnica de la revisión sistemática permite resumir información sobre una realidad de manera minuciosa y empírica, obteniendo una conclusión general a partir del estudio de trabajos individuales (Gómez et al., 2014). El uso de esta técnica ha posibilitado el estudio del impacto real en la producción científica, en cuanto a uso y aplicación, del marco competencial Entrecomp en trabajos del ámbito de las competencias emprendedoras y la posibilidad de su entrenamiento para el emprendimiento. Esta revisión se ha realizado tomando como referencia a Gisbert y Bonfill (2004). El conjunto de tareas y procesos necesarios para establecer los trabajos objeto de estudio se encuentran reflejados en el diagrama de flujo de la figura 2.

Las bases de datos seleccionadas las consideradas en la actualidad como más relevantes en la comunidad científica (Cadavid Higuita et al., 2012). Se han analizado trabajos procedentes de (por orden alfabético): 1.ABI-Inform/ProQuest (ABI. Inf. P.Q), 2.- Dialnet, 3.- Latindex, 4.- Redalyc, 5.- Scielo, 6.- Science Direct, 7.Scopus y 8.- Web of Science (WoS). El espacio temporal ha sido 1 de enero de 2017 (Entrecomp fue publicado en noviembre de 2016) y el 31 de julio de 2019. Para acotar el análisis a aquellos trabajos en los que el proceso de revisión sea lo más exigente y restrictivo posible (Liao et al., 2017) se han excluido los tipos de documentos: 1) libro, 2) capítulo de libro, 3) comunicaciones en conferencias y congresos y 4) material divulgativo relacionado.

Para establecer las palabras clave, se partió de los campos genéricos "emprendimiento" y "competencias emprendedoras". El campo específico fue "marco competencial Entrecomp". El uso de estas palabras vino determinado por el interés en identificar aquellos trabajos que relacionaban el emprendimiento y las competencias emprendedoras con el uso y aplicación del marco competencial emprendedor Entrecomp. La búsqueda se realizó en inglés. En el caso de los campos genéricos, "entrepreneurship" y "entrepreneurial competencies" y "entrepreneurship competence framework Entrecomp" para el campo específico. La consulta se realizó en este idioma ya que todas las bases de datos consultadas, sea cual sea la lengua de publicación del trabajo, disponen del resumen y las palabras clave en inglés. Esto facilitaba el acceso de manera homogénea a la totalidad de trabajos de interés para el estudio.

Las consultas en las distintas de bases arrojó un primer resultado de 43 trabajos. Estas referencias fueron exportadas al gestor bibliográfico Mendeley de Elsevier. Una vez se integró la búsqueda, se excluyeron aquellos trabajos que no eran artículos (Cornelius et al., 2006) junto con aquellos que estaban duplicados (Conde y Tercedor, 2015). El resultado final fue de 23 artículos objeto de estudio. Los otros 20 trabajos fueron desechados del estudio con el fin de favorecer confusiones y errores en el tratamiento de los datos. Este proceso se observa de manera más detallada en la figura 2.

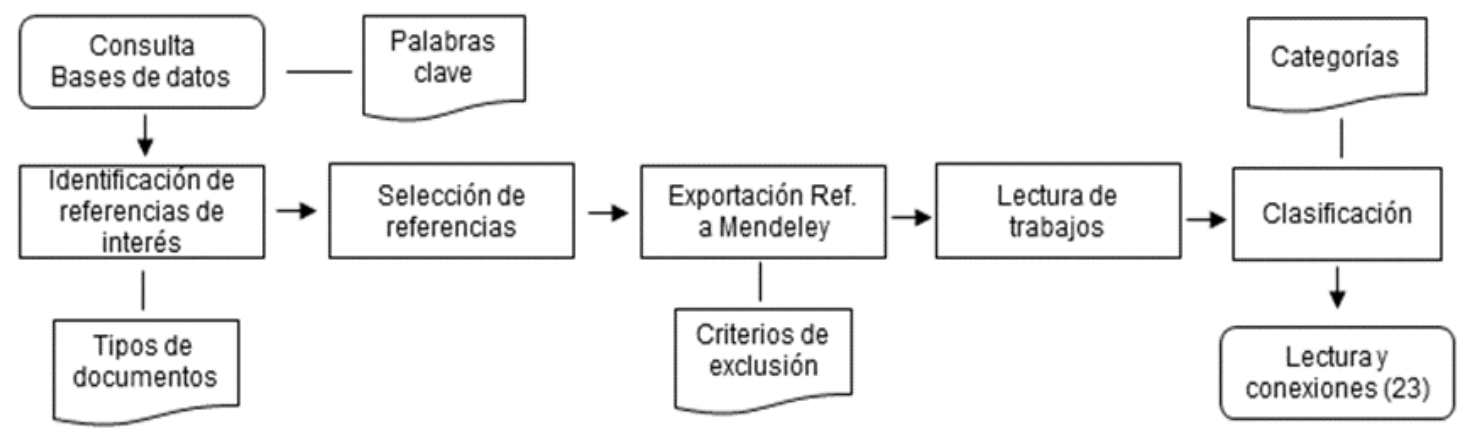

Fig. 2. Diagrama de flujo para la selección de trabajos

Las 23 referencias fueron leídas por 2 de los autores para su clasificación. En caso de duda, un tercer autor leyó el artículo para resolverla. Como resultado de este análisis se clasificaron las 23 referencias en siete categorías: 1) tipo de trabajo: 1.1) empíricos, 1.2) estudios de caso, 1.3) revisiones de literatura o 1.4) teóricos. 2) año de publicación. 3) país de filiación de los/as autores/as. 4) idioma empleado 5) palabras clave, 6) revista de publicación y 7) objetivos. Una vez clasificados los articulos, se procedió de nuevo a su lectura a fin de establecer los temas clave abordados y posibles conexiones entre los distintos trabajos objeto de estudio. El listado completo de los trabajos (43) y su clasificación, está disponible por parte de los autores bajo petición.

\section{RESULTADOS}

Una vez analizados los 23 artículos y según la clasificación expuesta en la metodología, los primeros resultados obtenidos fueron los siguientes: 1) tipo de trabajo. 1.1) empíricos: 9, 1.2) estudios de caso: 2, 1.3) revisiones de literatura: 1 y 1.4) teóricos: 11 . En cuanto al año de publicación, hay que destacar que Entrecomp es publicado en noviembre de 2016 por lo que el horizonte temporal de este trabajo se circunscribe a un total de 4 posibles años. No es hasta el año 2017 cuando se identifican las primeras menciones a Entrecomp. Concretamente en 2017 son 9 los trabajos en los que se hace referencia, 6 en 2018 y 8 en 2019. 
Tabla 2. Cuadro-resumen artículos, base de datos de acceso y objetivos.

\begin{tabular}{|c|c|c|}
\hline Artículo & Base de datos & Objetivo del trabajo \\
\hline (Palos-Sánchez et al., 2019) & $\begin{array}{l}\text { WoS/ Scopus/ } \\
\text { Redalyc/ Dialnet/ ABI. } \\
\text { Inf. P. Q }\end{array}$ & $\begin{array}{l}\text { Clasificar las competencias emprendedoras de las personas } \\
\text { usuarias del programa Diagnóstico Emprende de la J. de } \\
\text { Extremadura (España) a partir de Entrecomp. }\end{array}$ \\
\hline (Dinning, 2019) & Scopus/ ABI. Inf. P.Q & $\begin{array}{l}\text { Explorar el nivel de uso del lenguaje emprendedor en la } \\
\text { documentación de programa y como el currículum es } \\
\text { sustentado sobre las competencias emprendedoras. Toma } \\
\text { como referencia Entrecomp. }\end{array}$ \\
\hline (Strauti et al., 2018) & Science Direct & $\begin{array}{l}\text { Analizar el plan de estudios actual para el grado de } \\
\text { Ingeniería económica desde la perspectiva Entrecomp. }\end{array}$ \\
\hline (Fagerholm et al., 2018) & Science Direct & $\begin{array}{l}\text { Establecer los patrones y anti-patrones de un programa } \\
\text { basado en experiencias y proyectos. Los resultados son } \\
\text { confrontados con Entrecomp. }\end{array}$ \\
\hline (Lynch et al., 2019) & Science Direct & $\begin{array}{l}\text { Explorar el uso de la metodología Design Thinking como vía } \\
\text { para favorecer el desarrollo del espíritu emprendedor entra } \\
\text { las personas participantes. }\end{array}$ \\
\hline (Paños-Castro, 2017) & Redalyc & $\begin{array}{l}\text { Establecer a través de una revisión bibliográfica el término } \\
\text { emprender. Habilidades y destrezas que forman parte de } \\
\text { esta competencia y cuál es el objetivo de la educación } \\
\text { emprendedora. }\end{array}$ \\
\hline (Aurik y Astri, 2018) & ABI. Inf. P.Q & $\begin{array}{l}\text { Analizar y comprar el nivel de competencias emprendedoras } \\
\text { del alumnado/a de dos instituciones educativas participantes } \\
\text { en programas de educación emprendedora }\end{array}$ \\
\hline (Lilleväli y Täks, 2017) & ABI. Inf. P.Q & $\begin{array}{l}\text { Comprender el desarrollo de la competencia emprendedora } \\
\text { en todos niveles educativos y como se conceptualizan en los } \\
\text { diferentes modelos competenciales de educación } \\
\text { emprendedora. }\end{array}$ \\
\hline (Cavallini et al., 2019) & ABI. Inf. P.Q & $\begin{array}{l}\text { Analizar en los estudios de máster universitario el impacto } \\
\text { de la educación emprendedora. }\end{array}$ \\
\hline $\begin{array}{l}\text { (Fiore, Sansone, y Paolucci, } \\
\text { 2019) }\end{array}$ & ABI. Inf. P.Q & $\begin{array}{l}\text { Mostrar la importancia de crear equipos con diferentes } \\
\text { competencias, habilidades cognitivas y de toma de } \\
\text { decisiones. }\end{array}$ \\
\hline (Gedeon, 2017) & ABI. Inf. P.Q & $\begin{array}{l}\text { Describir cómo medir la transformación del alumnado tras } \\
\text { participar en un programa de emprendimiento en un grado } \\
\text { universitario. }\end{array}$ \\
\hline (Beyhan y Findik, 2018) & ABI. Inf. P.Q & $\begin{array}{l}\text { Investigar los vínculos entre las competencias organizativas } \\
\text { a nivel universitario, las competencias emprendedoras de } \\
\text { los empresarios incipientes y el número de empresas de } \\
\text { nueva creación creadas por estudiantes y recién graduados. }\end{array}$ \\
\hline (Pawitan et al., 2018) & ABI. Inf. P.Q & $\begin{array}{l}\text { Mostrar la relación entre las competencias emprendedoras } \\
\text { de las personas estudiantes y las competencias de éstos } \\
\text { para comprender procesos de productivos y de alta } \\
\text { tecnología. }\end{array}$ \\
\hline (Lindner, 2018) & ABI. Inf. P.Q & $\begin{array}{l}\text { Proporcionar información sobre la educación para el } \\
\text { emprendimiento, con un aprendizaje basado en el desafío } \\
\text { empresarial para un futuro sostenible. }\end{array}$ \\
\hline (Niccum et al., 2017) & ABI. Inf. P. Q & $\begin{array}{l}\text { Caracterizar la educación en innovación y emprendimiento } \\
\text { de las escuelas de medicina alopática de EE. UU. }\end{array}$ \\
\hline (Fleacă, 2017) & ABI. Inf. P.Q & $\begin{array}{l}\text { Propone un modelo basado en procesos para integrar y } \\
\text { aprovechar las tecnologías de aprendizaje digital en la } \\
\text { enseñanza, el aprendizaje y las prácticas organizativas. }\end{array}$ \\
\hline (Colin, 2019) & ABI. Inf. P.Q & $\begin{array}{l}\text { Identificar una firma pedagógica para el emprendimiento que } \\
\text { se pueda utilizar en todos los contextos de enseñanza y } \\
\text { aprendizaje asociados con todas las formas de educación } \\
\text { emprendedora. }\end{array}$ \\
\hline (Draksler y Širec, 2019) & ABI. Inf. P.Q & $\begin{array}{l}\text { Formular un modelo de investigación único y construcción } \\
\text { de un instrumento de medición personalizado para el estudio } \\
\text { del impacto de la educación empresarial en las } \\
\text { competencias e intenciones emprendedoras. }\end{array}$ \\
\hline (Hatt, 2018) & ABI. Inf. P.Q & $\begin{array}{l}\text { Presentar resultados sobre la perspectiva de los } \\
\text { emprendedores sobre conceptos críticos para pensar como } \\
\text { persona emprendedora. }\end{array}$ \\
\hline
\end{tabular}


Tabla 2: (continuación)

\begin{tabular}{|l|l|l|}
\hline Artículo & Base de datos & Objetivo del trabajo \\
\hline $\begin{array}{l}\text { (Camacho-Miñano y del } \\
\text { Campo, 2017) }\end{array}$ & ABI. Inf. P.Q & $\begin{array}{l}\text { Analizar el nivel de creatividad de los estudiantes de } \\
\text { administración de empresas asistentes a un seminario de } \\
\text { emprendimiento en contraste con los que no. }\end{array}$ \\
\hline (Arruti y Paños Castro, 2019) & ABI. Inf. P.Q & $\begin{array}{l}\text { Profundizar en la formación inicial del profesor/a } \\
\text { emprendedor/a del grado en Educación Primaria, a través } \\
\text { del análisis de las menciones ofertadas en el grado en } \\
\text { Educación Primaria en España }\end{array}$ \\
\hline (Hatzijordanou et al., 2019) & ABI. Inf. P.Q & $\begin{array}{l}\text { Determinar el estado de la investigación sobre el análisis de } \\
\text { la competencia como piedra angular de la gestión } \\
\text { estratégica. }\end{array}$ \\
\hline (Azqueta, 2019) & $\begin{array}{l}\text { Conocer el origen y evolución etimológica del término } \\
\text { emprendedor y analizar la tradición intelectual y las } \\
\text { principales teorías que recoge el concepto. }\end{array}$ \\
\hline
\end{tabular}

Con relación a las palabras clave empleadas, es necesario hacer una mención especial sobre 3 de los 23 artículos, ya que estos no especificaban ninguna. En concreto, ocurre en los artículos An analysis of differences in students' entrepreneurial competencies between the management and entrepreneurship study programmes at the school of business and management (Sbm) institut teknologi bandung (itb) de Aurik y Astri (2018), Competence Models as a Tool for Conceptualizing the Systematic Process of Entrepreneurship Competence Development de Lilleväli y Täks (2017) y Measuring Student Transformation in Entrepreneurship Education Programs de Gedeon (2017). En los demás trabajos, 20 en total, sí incluían todos palabras clave. En total, son 67 palabras clave distintas sobre 94 palabras clave totales.

Tabla 3. Revistas y publicaciones por años

\begin{tabular}{|c|c|c|c|c|}
\hline Revista & 2017 & 2018 & 2019 & Total \\
\hline Administrative Sciences & - & - & 2 & 2 \\
\hline Discourse and Communication for Sustainable Education & - & 1 & - & 1 \\
\hline Education + Training & 2 & - & - & 2 \\
\hline Education Research International & 2 & - & - & 2 \\
\hline Interciencia & - & - & 1 & 1 \\
\hline Journal of Entrepreneurship Education & - & 2 & 1 & 3 \\
\hline Journal of Small Business and Enterprise Development & - & - & 1 & 1 \\
\hline Journal of Technology Transfer & 1 & - & - & 1 \\
\hline Journal System and Software & - & 1 & - & 1 \\
\hline Management Review Quarterly & - & - & 1 & 1 \\
\hline Medical Education Online & 1 & - & - & 1 \\
\hline Nase Gospodarstvo & - & 1 & - & 1 \\
\hline Procedia - Social and Behavioral Sciences & - & 1 & - & 1 \\
\hline Revista Complutense de Educación & 1 & - & - & 1 \\
\hline $\begin{array}{l}\text { Revista Electrónica Interuniversitaria de Formación del } \\
\text { Profesorado }\end{array}$ & 1 & - & - & 1 \\
\hline Technological Forecasting and Social Change & - & - & 1 & 1 \\
\hline TEM Journal & 1 & - & & 1 \\
\hline Teoría de la Educación; Revista Interuniversitaria Salamanca & - & - & 1 & 1 \\
\hline
\end{tabular}

Respecto a la filiación de los/as autores/as, son 15 países distintos, los que aparecen referenciados en los 23 trabajos analizados. En concreto, filiaciones procedentes de 9 países de la UE. 2 pertenecientes a países del continente europeo, pero no de la UE y 4 del resto del mundo. Inglaterra sigue perteneciendo a fecha de agosto de 2019 a la UE hasta que su salida se formalice oficialmente. El idioma de publicación ha sido en su gran mayoría, en inglés. Solo 4 han sido publicados en otro idioma, en concreto en español. 
Sobre la filiación de los trabajos analizados, destaca como, siendo Entrecomp un marco competencial que surge en la UE, los trabajos científicos que lo abordan no todos tienen necesariamente filiación en ésta. Países no pertenecientes a la UE, pero sí cercanos geográficamente como son Noruega y Turquía han realizado trabajos donde se menciona. Incluso en países más lejanos y diferentes tanto a nivel cultural como educativo como Australia, Canadá, Estados Unidos e Indonesia, se han identificado autores con filiaciones de estos países. Destaca el caso de autores con filiación española, siendo el país con mayor número de trabajos, 5 , y en 4 de ellos publicados en idioma español. Esta situación muestra sin duda el interés desde la comunidad investigadora española sobre Entrecomp, como materia de estudio y análisis. Con respecto a las bases de carácter científico en el que trabajos que usan o aplican Entrecomp, destaca como, en el caso de las consideradas de mayor impacto entre la comunidad científica en la actualidad: WoS, Scopus y Scielo la presencia de trabajos identificados sea baja. En el caso de WoS, hay sólo 1 trabajo. En el caso de Scopus se identifican solo 2 y en Scielo ninguno. La base de datos en la que se ha identificado un mayor número de trabajos objeto de estudio es ABI Information ProQuest, con un total de 19.

\section{DISCUSIÓN}

A pesar de lo relevante de la temática abordada a través de Entrecomp y del objetivo de la UE con su publicación, su impacto dentro de la literatura científica es limitada. Sobre el tipo de aplicación que tiene, destaca como, en 9 trabajos, éste sirve como base para la realización de trabajos de carácter empírico. Trabajos en los que las competencias de este marco son utilizadas como referencia para analizar distintos colectivos y ámbitos relacionados con la educación emprendedora.

Otro de los aspectos relevantes dentro de los resultados obtenidos, es el hecho del estudio de las palabras clave utilizadas. Cómo se observa, las directamente relacionadas con el ámbito de las competencias emprendedoras, son las que tienen un mayor número de repeticiones. Éstas aportan información sobre temáticas vinculadas a trabajos sobre emprendimiento y educación. Las palabras son entrepreneurship education, entreprenurial competences, entrepreneurship, curriculum, design thinking, entrecomp (framework), entreprenurial intention, higher education, entrepreneur y University. Algo similar ocurre en el caso de las revistas en las que han sido publicados estos trabajos. Destacan revistas relacionadas con la educación las que tienen mayor número de artículos publicados, concretamente: Journal of Entrepreneurship Education, Education Research International, Education + Training y Administrative Sciences. Quedando evidenciado como a pesar de lo limitado de su uso sí que está relacionado con los ámbitos y áreas de conocimiento afines según su objeto.

Finalmente, respecto a las temáticas abordadas en estos trabajos, tal y cómo se observa en la tabla 2, en el caso de los trabajos empíricos, Entrecomp, ha sido aplicado a la hora de establecer las competencias emprendedoras como base para la obtención de resultados. En el resto de los trabajos, este marco ha sido abordado a la hora de analizar y/o mencionar éste como un marco posible y viable a la hora de establecer competencias para el emprendimiento, pero sin profundizar mucho más. No hay en la actualidad ningún artículo que examine o reflexione sobre la viabilidad de cada uno de los elementos que componen Entrecomp. Al tratarse este trabajo de una revisión literaria de una realidad aún reciente, no existen otros trabajos anteriores en los que se aborde el impacto en la literatura científica de Entrecomp. No es posible por tanto comparar y establecer puntos de conexión con acuerdo o desacuerdo respecto a los datos obtenidos y su interpretación llevados a cabo en éste.

\section{CONCLUSIONES}

De la revisión sistemática de la literatura, de su análisis y de su discusión, se pueden obtener las siguientes conclusiones en este trabajo: 1) a fecha 2019, Entrecomp tiene un limitado impacto en la producción científica relacionada con las competencias emprendedoras y su entrenamiento. Es un marco enunciado por un organismo supranacional europeo cuyo objetivo es marcar la pauta a los gobiernos de los países miembros en el favorecimiento de políticas emprendedoras en los distintos niveles educativos. 2) Hay una escasa conexión, a tenor de los trabajos analizados y los resultados obtenidos, entre la investigación científica relacionada con Entrecomp y el desarrollo de políticas en favor del emprendimiento, las competencias emprendedoras y su entrenamiento por parte de los poderes públicos implicados, a pesar de ser este uno de sus objetivos. 3) Dentro del escaso impacto en la literatura científica, a pesar de ser Entrecomp una iniciativa europea, el uso y aplicación de este marco competencial no se circunscribe a trabajos solo de países de la UE.

\section{REFERENCIAS}

Arruti, A., y Paños, J., Análisis de las menciones del grado en Educación Primaria desde la perspectiva de la competencia emprendedora. https://doi.org/10.5209/RCED.55448. Revista Complutense de Educación, 30(1), 17-33 (2019). 
Aurik, G., y Astri, G., An analysis of differences in students' entrepreneurial competencies between the management and entrepreneurship study programmes at the school of business and management (Sbm) institut teknologi bandung (itb). https://doi.org/10.5539/ies.v7n12p1, Journal of Entrepreneurship Education, 21(4), 1-11 (2018).

Azqueta, A., Análisis del concepto emprendedor y su incorporación al ámbito educativo. http://dx.doi.org/10.14201/teri.19756, Ediciones Universidad de Salamanca, 31(1), 57-80 (2019).

Bacigalupo, M., Kampylis, P., Punie, Y. y Van den Brande, G., EntreComp: The Entrepreneurship Competence Framework. https://doi.org/10.2791/593884, Publication Office of the European Union. Luxembourg (2016).

Beyhan, B., y Findik, D., Student and graduate entrepreneurship: ambidextrous universities create more nascent entrepreneurs. https://doi.org/10.1007/s10961-017-9590-z, Journal of Technology Transfer, 43(5), 1346-1374 (2018).

Cadavid, L., Awad, G., y Franco, C.J., Análisis bibliométrico del campo modelado de difusión de innovaciones. https://doi.org/10.18046/j.estger.2012.1486, Estudios Gerenciales, 28(65), 213-236 (2012).

Camacho-Miñano, M. D. M., y del Campo, C., The role of creativity in entrepreneurship: an empirical study on business undergraduates. https://doi.org/10.1108/ET-08-2016-0132, Education and Training, 59(7-8), 672-688 (2017).

Cavallini, F., y otros 4 autores, Allenamente: the Case of the Aba Master At the University of Studies of Parma. ISSN: 1528-2651, Entrepreneurship Education, 22(1), 1-13 (2019).

Colin, J., A signature pedagogy for entrepreneurship education. https://doi-org.libproxy1.nus.edu.sg/10.1108/JSBED-032018-0080, Journal of Small Business and Enterprise Development, 26(2), 243-254 (2019).

Conde, M. A., y Tercedor, P., La actividad física, la educatión física y la conditión física pueden estar relacionadas con el rendimiento académico y cognitivo en jóvenes. ISSN 0212-8799, Arch. de Medicina Del Deporte, 32(2), 100-109 (2015).

Cornelius, B., Landström, H., y Persson, O., Entrepreneurial Studies: The Dynamic Research Front of a Developing Social Science. https://doi.org/10.1111/j.1540-6520.2006.00125.x, Entrepreneurship Theory and Practice, 30(3), 375-398 (2006).

Davidsson, P., Entrepreneurial opportunities and the entrepreneurship nexus: $A$ re-conceptualization. https://doi.org/10.1016/j.jbusvent.2015.01.002, Journal of Business Venturing, 30(5), 674-695 (2015).

Dinning, T., Articulating entrepreneurial competencies in the undergraduate curricular. https://doi.org/10.1108/ET-09-20180197, Education + Training, 61(4), 432-444 (2019).

Draksler, T. Z., y Širec, K., Conceptual Research Model for Studying Students' Entrepreneurial Competencies. http://dx.doi.org/10.2478/ngoe-2018-0020, Naše Gospodarstvo/Our Economy, 64(4), 23-33 (2019).

Edwards-Schachter, M., García-Granero, A., Sánchez-Barrioluengo, M., Quesada-Pineda, H., y Amara, N., Disentangling competences: Interrelationships on creativity, innovation and entrepreneurship. https://doi.org/10.1016/j.tsc.2014.11.006, Thinking Skills and Creativity, 16, 27-39 (2015).

Fagerholm, F., Hellas, A., Luukkainen, M., Kyllönen, K., Yaman, S., y Mäenpää, H., Designing and implementing an environment for software start-up education: Patterns and anti-patterns. https://doi.org/10.1016/j.jss.2018.08.060, Journal of Systems and Software, 146, 1-13 (2018).

Fiore, E., Sansone, G., y Paolucci, E., Entrepreneurship Education in a Multidisciplinary Environment: Evidence from an Entrepreneurship Programme Held in Turin. https://doi.org/10.3390/admsci9010028, Administrative Sciences, 9(1), 28 (2019).

Fleacă, E., Entrepreneurial Curriculum through Digital-Age Learning in Higher Education - A Process-based Model. https://doi.org/10.18421, TEM Journal, 6(3), 591-598 (2017).

Gedeon, S. A., Measuring Student Transformation in Entrepreneurship Education Programs. https://doi.org/10.1155/2017/8475460, Education Research International, 2017, 1-12 (2017).

Gisbert, J. P., y Bonfill, X., ¿Cómo realizar, evaluar y utilizar revisiones sistemáticas y metaanálisis? https://doi.org/10.1157/13058397, Gastroenterologia y Hepatologia, 27(3), 129-149 (2004).

Gómez, G., Aguileta, A., Ancona, G.B., y Gómez, O.S., Avances en las Mejoras de Procesos Software en las MiPyMEs Desarrolladoras de Software: Una Revisión Sistemática. https://doi.org/10.18294/relais.2014.262-268, Revista Latinoamericana de Ingenieria de Software, 2(4), 262 (2014).

Hatt, L., Threshold concepts in entrepreneurship - the entrepreneurs' perspective. https://doi.org/10.1108/ET-08-20170119, Education and Training, 60(2), 155-167 (2018).

Hatzijordanou, N., Bohn, N., y Terzidis, O., A systematic literature review on competitor analysis: status quo and start-up specifics. https://doi.org/10.1007/s11301-019-00158-5, Management Review Quarterly (2019).

Jiménez, A., Palmero-Cámara, C., González-Santos, M.J., González-Bernal, J., y Jiménez-Eguizábal, J.A., The impact of educational levels on formal and informal entrepreneurship. https://doi.org/10.1016/j.brq.2015.02.002, BRQ Business Research Quarterly, 18(3), 204-212 (2015).

Liao, Y., Deschamps, F., Loures, E. de F.R., y Ramos, L.F.P., Past, present and future of Industry 4.0 - a systematic literature review and research agenda proposal. https://doi.org/10.1080/00207543.2017.1308576, International Journal of Production Research, 55(1), 1-21 (2017). 
Lilleväli, U., y Täks, M., Competence Models as a Tool for Conceptualizing the Systematic Process of Entrepreneurship Competence Development. https://doi.org/10.1155/2017/5160863, Education Research International, 2017, 1-16 (2017).

Lindner, J., Entrepreneurship Education for a Sustainable Future. https://doi.org/10.2478/dcse-2018-0009, Discourse and Communication for Sustainable Education, 9(1), 115-127 (2018).

Lynch, M., Kamovich, U., Longva, K. K., y Steinert, M., Combining technology and entrepreneurial education through design thinking: Students' reflections on the learning process. https://doi.org/10.1016/j.techfore.2019.06.015 Technological Forecasting and Social Change (2019).

McCallum, E., Weicht, R., McMullan, L., y Price, A., EntreComp into Action - Get inspired, make it happen: A user guide to the European Entrepreneurship Competence Framework. Luxembourg. https://doi.org/10.2760/574864, Publications Office of the European Union (2018).

Niccum, B. A., Sarker, A., Wolf, S.J. y Trowbridge, M.J., Innovation and entrepreneurship programs in US medical education: A landscape review and thematic analysis. https://doi.org/10.1080/10872981.2017.1360722, Medical Education Online, 22(1) (2017).

Palos-Sánchez, P. R., Baena-Luna, P. y Casablanca Peña, A., Análisis de las competencias educativas para evaluar a las personas emprendedoras. ISSN 0378-1844, Interciencia, 44(May), 291-297 (2019).

Paños-Castro, J. Educación emprendedora y metodologías activas para su fomento. https://doi.org/10.6018/reifop.20.3.272221, Revista Electrónica Interuniversitaria de Formación Del Profesorado, 20(3), 33-48 (2017).

Pawitan, G., Widyarini, M. y otros seis autores, The competence of young entrepreneur candidate in university, Indonesia. ISSN: 1528-2651, Education and Training, 60(3), 468-482 (2018).

Premand, P., Brodmann, S., Almeida, R., Grun, R. y Barouni, M., Entrepreneurship Education and Entry into SelfEmployment Among University Graduates. https://doi.org/10.1016/j.worlddev.2015.08.028, World Development, 77, 311327 (2016).

Rengamani, J., y Shameem, A., Inevitability of the entrepreneurial skills among the mechanical engineering students in Chennai - an empirical study. ISSN 0976-6359, International Journal of Mechanical Engineering and Technology, 9(7), 1455-1463 (2018).

Sanchis, R., Poler R., y Ortiz, A., Técnicas para el Modelado de Procesos de Negocio en Cadenas de Suministro. https://doi.org/10.4067/S0718-07642009000200005, Información Tecnológica, 20(2), 29-40 (2009).

Soria-Barreto, K., Zuniga-Jara, S. y Ruiz-Campo, S., Educación e intención emprendedora en estudiantes universitarios: Un caso de estudio. https://doi.org/10.4067/S0718-50062016000100004, Formacion Universitaria, 9(1), 25-34 (2016).

Strauti, G., Dumitrache, V.M. y Taucean, I.M., Entrepreneurial Competences in Economical Engineering Curriculum in Romania. https://doi.org/10.1016/j.sbspro.2018.04.057, Procedia - Social and Behavioral Sciences, 238, 737-742 (2018).

Suddaby, R., Bruton G.D., y Si, S.X., Entrepreneurship through a qualitative lens: Insights on the construction and/or discovery of entrepreneurial opportunity. https://doi.org/10.1016/j.jbusvent.2014.09.003, Journal of Business Venturing, 30(1), 1-10 (2015). 